\title{
MANIFESTAÇÕES DERMATOLÓGICAS EM PACIENTES COM COVID-19
}

\author{
*Rafaella Fiquene de Brito Filgueira I \\ Estephanye Vasconcelos Nunes de Farias I \\ Thássia Rachel Brito de Figueiredo de Almeidal \\ Maria Eduarda Rodrigues Castelianno "
}

\section{RESUMO}

A COVID-19, uma doença causada pelo novo coronavírus, o SARS-CoV-2, surgiu na China em dezembro de 2019 e rapidamente começou a se espalhar por todo o mundo. Porém, os sintomas são muito inespecíficos e variáveis, dificultando o diagnóstico clínico da doença. Diante disso, o presente trabalho tem o objetivo de analisar a ocorrência das manifestações cutâneas e seus tipos nos pacientes portadores de COVID-19. Trata-se de uma revisão integrativa, realizada em outubro de 2020, cujas questões norteadoras foram: <Manifestação dermatológica em pacientes com COVID-19 pode ser método diagnóstico de paciente com coronavírus? E, quais manifestações e complicações estão relacionadas a COVID-19?>. Apenas em março de 2020, os primeiros estudos sobre manifestações dermatológicas foram publicados, mostrando que os pacientes, em sua maioria, não apresentam lesões cutâneas e, os que apresentam, têm majoritariamente erupção eritematosa. No entanto, foram relatados os mais diferentes tipos de lesões. Devido a dificuldade em fazer o seguimento dos portadores das infecções por coronavírus, ainda não é conhecido seu mecanismo. O que se sabe é que a afecção é auto-limitada e que algumas drogas podem induzir a sua formação, dificultando a diferenciação entre manifestação do distúrbio e do tratamento. Sendo assim, as condutas devem ser individualizadas de modo a trazer uma melhor resposta terapêutica e entendimento da patologia. É importante estar atento principalmente aos enfermos que fazem uso de terapia imunossupressora.

PALAVRAS-CHAVE: Coronavírus. Manifestações Cutâneas. Infecções por Coronavírus.

Graduandas. Faculdade de Medicina Nova Esperança Esperança, FAMENE. Departamento de MEDICINA. I CEP: 58067-698, João Pessoa, Paraíba, Brasil. *Autor correspondente: rafilgueira@gmail.com. ORCID ID: 0000-0003-2035-0684; 0000-0002-6223-8052; 0000-0002-6223-8052.

Médica graduada pela Faculdade de Medicina Nova Esperança, FAMENE. CEP: 58067-698, João Pessoa, Paraíba, Brasil. Orcid: 0000-0003-3082-4417 


\section{INTRODUÇÃO}

Um tipo de pneumonia diferente começou a circular em dezembro de 2019 em Wuhan, na China. ${ }^{1}$ O que levou a descoberta de uma doença causada pelo novo coronavírus humano, SARS-CoV-2. Essa patologia se disseminou por todo o mundo em um curto espaço de tempo e foi denominada COVID-19. Apenas em março de 2020 foi caracterizada pela Organização Mundial da Saúde (OMS) como uma pandemia. ${ }^{2}$

As infecções respiratórias agudas (IRA) são um constante desafio para a saúde pública mundial, sendo importantes causas de morbimortalidade em todo o mundo. ${ }^{1}$ Já a Síndrome Respiratória Aguda (SARS) tem uma alta morbimortalidade, até o dia 20 de outubro de 2020, sendo confirmados 40.551.950 casos no mundo e 1.123.128 mortes. No Brasil, 5.274.817 casos foram confirmados, com 154.888 óbitos nessa mesma data, sendo um dos países com maior número de casos em todo o mundo. 3

As manifestações dermatológicas foram relatadas em vários pacientes, sendo a urticária e a hipersensibilidade a medicamentos os sintomas cutâneos mais frequentes. Ainda é muito difícil diferenciar a reação medicamentosa de um sintoma da doença, já que esses pacientes são comumente tratados com antibióticos e antivirais que podem causar essas erupções na pele, sendo importante observar essas manifestações e fazer o diagnóstico diferencial precoce. 4

Além da urticária, esses pacientes também podem desenvolver erupção cutânea mobiliforme, erupções vesiculares, lesões "acrais", erupções do tipo livedo reticular e outras erupções cutâneas incomuns. É importante observar e diferenciar as manifestações dermatológicas, pois o mesmo paciente pode apresentar múltiplas anormalidades simultâneas na pele que diferem na morfologia. 5

Pacientes graves com COVID-19 podem apresentar um estado de Coagulação Intravascular Disseminada (CIVD), apresentando acroisquemia com cianose dos dedos das mãos e dos pés, gangrena seca e bolhas cutâneas. ${ }^{6}$ Esses pacientes apresentam um maior risco de eventos tromboembólicos, logo aumentando o risco de óbitos. 7

Os pacientes podem ainda ser assintomáticos ou desenvolverem sintomas leves. Os sintomas comumentes encontrados são: febre, dispneia e tosse seca, dores, congestão nasal, erupção cutânea ou descoloração dos dedos das mãos ou dos pés, dor de cabeça, conjuntivite, dor de garganta, perda de paladar ou olfato. ${ }^{8}$

Apesar das manifestações serem majoritariamente leves ou assintomáticas, cerca de $20 \%$ dos pacientes precisam de tratamento hospitalar, e 1 a cada 6 pacientes ficam enfermos com dificuldade respiratória e necessitando de internação por terem maior gravidade de doença. Idosos e pessoas com algumas comorbidades têm maiores riscos de ficarem gravemente doentes. 3 Embora, muito se tenha estudado e pesquisado sobre a doença, o diagnóstico clínico não é possível devido ao desconhecimento e a variabilidade dos sintomas. Dessa forma, todos os pacientes precisam de exames laboratoriais para confirmar a doença.

Diante do exposto, o presente trabalho tem o objetivo de analisar a ocorrência das manifestações cutâneas e seus tipos em pacientes portadores de COVID-19. 


\section{MATERIAL E MÉTODOS}

Trata-se de uma revisão integrativa, realizada em outubro de 2020 . Foi dividida em 4 etapas:

Primeira etapa: definição do tema e questões norteadoras <Manifestação dermatológica em pacientes com covid-19 pode ser método diagnóstico de paciente com coronavírus? E quais manifestações e complicações dermatológicas estão relacionadas ao COVID-19? >;

Segunda etapa: para a composição da revisão de literatura científica especializada, foi feita uma pesquisa mediante busca nas bases LILACS e PUBMED a partir do uso dos descritores e booleanos: <Dermatology AND Manifestations AND COVID-19>; Dermatologic AND COVID-19 AND Complications>. Desse modo, foram encontrados 28 (LILACS) 32 (PUBMED) artigos, utilizando os descritores e booleanos < Dermatology AND Manifestations AND COVID-19>; e, 32 e 10 artigos no LILACS e PUBMED, respectivamente, utilizando os descritores e booleanos: <Dermatologic AND COVID-19 AND Complications> (Tabela 1).

TABELA 1: Distribuição dos artigos encontrados nas bases de dados LILACS e PUBMED, usando os descritores e booleanos: <Dermatology AND Manifestations AND COVID-19>; Dermatologic AND COVID-19 AND Complications>.

\begin{tabular}{ccc}
\hline \multirow{2}{*}{ DESCRITORES } & \multicolumn{2}{c}{ BANCO DE DADOS } \\
\cline { 2 - 3 } & LILACS & PUBMED \\
\hline Dermatology AND Manifestations AND COVID-19 & 28 & 32 \\
Dermatologic AND COVID-19-19 AND Complications & 23 & 10 \\
\hline & & Fonte: Os autores (2020)
\end{tabular}

Terceira etapa: foram utilizados os critérios de inclusão e exclusão para seleção dos artigos. Os critérios de inclusão foram: artigos disponíveis na íntegra na internet que abordassem o tema proposto, publicados no ano de 2020, revisões sistemáticas, metaanálises, revisões e estudos clínicos. Foram excluidos artigos, cujos títulos e resumos não se enquadravam na questão norteadora, artigos duplicados e recursos não científicos, sendo selecionados 8 PUBMED e 2 LILACS.

Quarta etapa: nessa última etapa foi realizada leitura na íntegra e elaboração da revisão.

\section{RESULTADOS E DISCUSSÃO}

Foram selecionados 10 artigos para compor a revisão. Os artigos desta revisão têm origem de diferentes países como: Itália (2), Nova Zelândia (1), Estados Unidos (3), Estados Unidos + Polônia (1), Bulgária (1 ), França (1) e Espanha (1) ( Tabela 2).

Em março de 2020, Recalti9 publicou a primeira perspectiva sobre manifestações dermatológicas do COVID-19, em que de 88 pacientes, 18 apresentaram manifestações dermatológicas. Destes, 8 pacientes desenvolveram manifestações no início da doença e 10 após internação. 14 pacientes apresentaram erupção eritematosa, 3 urticárias generalizadas e 1 vesículas semelhantes a varicela. A região mais 
comum era tronco e não foi evidenciado prurido significante. Não foi correlacionado a gravidade da doença e essas manifestações extra pulmonares desapareceram após alguns dias.

TABELA 2: Disposição dos artigos selecionados para compor a revisão integrativa, publicados no ano de 2020, considerando título, autores, país e banco de dados.

\begin{tabular}{|c|c|c|c|c|}
\hline ARTIGO & TíTULO & AUTORES & PAís & $\begin{array}{l}\text { BANCO DE } \\
\text { DADOS }\end{array}$ \\
\hline A9 & $\begin{array}{l}\text { Cutaneous manifestations in COVID-19: } \\
\text { a first perspective }\end{array}$ & Recalcati, S & Itália & PUBMED \\
\hline A10 & Extrapulmonary manifestations of COVID-19 & Gupta et al. & Estados Unidos & PUBMED \\
\hline A11 & $\begin{array}{c}\text { Cutaneous Manifestations of COVID-19: An Evidence-Based } \\
\text { Review }\end{array}$ & Daneshgaran et al. & Estados Unidos & LILACS \\
\hline $\mathrm{A} 12$ & $\begin{array}{l}\text { Chilblainlike lesions during COVID-19 epidemic: a preliminary } \\
\text { study on } 63 \text { patients }\end{array}$ & Piccolo et al. & Itália & PUBMED \\
\hline A13 & $\begin{array}{l}\text { Chilblains is a common cutaneous finding during the COVID-19 } \\
\text { pandemic: A retrospective nationwide study from France }\end{array}$ & Masson et al. & França & PUBMED \\
\hline A14 & $\begin{array}{l}\text { Chilblain-like lesions in pediatrics dermatological outpatients } \\
\text { during the COVID-19 outbreak }\end{array}$ & Garcia-Lara et al. & Espanha & PUBMED \\
\hline A15 & Cutaneous manifestations of COVID-19: a preliminary review & Jia et al. & Estados Unidos & PUBMED \\
\hline $\mathrm{A} 16$ & $\begin{array}{l}\text { Covid-19 pandemic and the skin-What should dermatologists } \\
\text { know? }\end{array}$ & Darlenski \& Tsankov & Bulgária & PUBMED \\
\hline A17 & $\begin{array}{l}\text { Advice regarding COVID-19 and use of immunomodulators, in } \\
\text { patients with severe dermatological diseases }\end{array}$ & Rademaker et al. & Nova Zelândia & PUBMED \\
\hline $\mathrm{A} 18$ & $\begin{array}{l}\text { Case Report: Chilblains-like lesions (COVID-19 toes) during the } \\
\text { pandemic - is there a diagnostic window? }\end{array}$ & Witkowski et al. & $\begin{array}{l}\text { Polândia/Estados } \\
\quad \text { Unidos }\end{array}$ & LILACS \\
\hline
\end{tabular}

Outro estudo mostrou lesões em forma de eritema pérnio, como manifestação mais comum, sendo relacionado a uma forma menos grave de COVID-19. ${ }^{10}$ Também relatado no estudo de Daneshgaran, ${ }^{11}$ que mostrou que esses pacientes com essas lesões normalmente não apresentavam sintomas e eram suspeitos devido a algum contato com paciente positivado para COVID-19. Assim, a detecção dessa lesão pode ser útil para identificar indivíduos assintomáticos com maior probabilidade de transmitir a doença.

No entanto, lesões em forma de livedos e necróticas foram associadas a uma maior gravidade da doença ${ }^{10}$, o que também foi encontrado no estudo de Daneshgaran, ${ }^{11}$ sendo as mais relacionadas a admissões hospitalares eventilação mecânica. Logo, pode ser que elas representem uma complicação específica de COVID-19, demonstrando a importância de observar essas lesões, suas mudanças e evoluções, pois podem ser muito úteis para o discutir prognóstico do paciente.

Através de observações, Picollo et al. ${ }^{12}$ notaram também o aumento de lesões "chilblainlike", eritema pérnio, na Itália concomitantemente com o surto do COVID-19, corroborando com o estudo de Gupta et al. ${ }^{10}$ Eritema pérnio pode ser primário, quando está relacionado a baixas temperaturas, e secundário, relacionado a doenças autoimunes, hematológicas e infecções virais, porém os pacientes, em geral, não apresentavam nenhuma dessas condições. 
Assim, pode-se inferir que esse surto teve relação com a pandemia pelo SARS$\mathrm{CoV}-2$, pois alguns pacientes positivaram e a maioria destes teve histórico familiar positivo para COVID-19. ${ }^{10,12}$

Esse mesmo estudo selecionou pacientes com lesões cutâneas e observou dois padrões em pacientes com lesões dolorosas ou pruriginosas em dedos e solas dos pés: eritematoso e edematoso; e formação de bolhas que se assemelham ao eritema pérnio. Contudo, não foram identificadas exposição prévia ao frio ou história pregressa desse quadro nesses pacientes, o que corrobora para sua associação a perniose. ${ }^{12}$

O estudo de Daneshgaran et al. ${ }^{11}$ teve a maioria dos pacientes confirmados laboratorialmente ou suspeitos de COVID-19. Nele, as lesões acrais foram o tipo mais encontradas, seguido por erupções cutâneas maculopapulares eritematosas, erupções vesiculares, erupções urticariformes e outros tipos de lesões, que se resolveram, em média, depois de 2-15 dias. Nos sintomáticos, a maioria tinha prurido, dor ou queimação. Embora possam ser encontradas em quase todas as faixas etárias, a média de idade de pacientes com erupções cutâneas vasculares foi de 77,5 anos e de lesões acrais em adultos jovens de 23,2 anos em média.

Masson et al. ${ }^{13}$ em um estudo observacional com 227 pacientes, maioria apresentando lesão acral, sendo as semelhantes a frieira as mais comuns, o que não é habitual na primavera na França. Embora a maioria não tenha se submetido ao teste de COVID-19, quase todos tiveram contato com pessoas portadoras ou apresentaram sintomas extracutâneos sugestivos. Estes estudos corroboram para a hipótese de associação do COVID-19 com esse tipo de lesão dermatológica, porém ainda não existem dados suficientes para provar a relação entre eles.
Outro estudo realizado em crianças e adolescentes com poucos sintomas ou assintomáticos, por Gárcia-Lara, ${ }^{14}$ evidenciou lesões acro-isquêmicas, semelhantes ao eritema pernioso nesses pacientes, localizados nos dedos das mãos e dos pés. Embora exista uma dificuldade para seguimento desses pacientes, dermatologistas, pediatras e clínicos devem estar cientes das lesões associadas à infecção por COVID-19 e suas possíveis complicações.

Casos de manifestações cutâneas relacionadas à vasculopatia em pacientes jovens saudáveis foram relatados, principalmente em crianças e adolescentes. Eles têm como característica máculas vermelho-violeta, que podem causar bolhas e, geralmente, evoluir favoravelmente. Lesões acroisquêmicas agudas e autocicatrizantes diferem da acrocianose, perniose ou vasculite, mas são decorrentes de danos endoteliais, isto é, microtromboses. Apesar de não ter implicação clínica bem definida, é primordial o monitoramento e o seu reconhecimento em pacientes mais jovens. ${ }^{14}$

A importância da estratificação de pacientes com manifestações cutâneas para COVID-19 é de suma importância, tendo em vista que muitos assintomáticos ou oligossintomáticos podem ser classificados erroneamente quanto ser portador ou não de coronavirus, e assim contaminar outras pessoas, sendo um potencial transmissor assintomático. ${ }^{15}$

As manifestações cutâneas do COVID-19 são auto-limitadas e ainda não se sabe ao certo como se dá o seu mecanismo. Pacientes com doenças relacionadas a pele que fazem uso de terapias biológicas têm seu uso interrompido, tendo em vista que ainda não se sabe se são possíveis outras complicações decorrentes do uso dessas medicações durante a vigência do COVID-19, sendo assim, toda conduta deve ser individualizada. 
Drogas como hidroxicloroquina, remdesivir, tocilizumabe devem ser analisadas antes de serem prescritas para pacientes com COVID-19 e com lesões na pele, tendo em vista que existe uma hipersensibilidade imune ao RNA do SARS-CoV-2, probabilidade de situações como vasculite, aumento liberações de citocinas e deposição de microtrombos. Nos exames histopatológicos das erupções cutâneas foram observados dermatite perivascular superficial e queratinócitos disqueratóticos. Nas biópsias das lesões acrocutâneas, foram identificados infiltrados linfóides difusos e densos, com inflamação endotelial associada. Foram observados nos vasos da derme formação de pequenos trombos. ${ }^{10}$

Para Darlenski et al., ${ }^{16}$ não existe um consenso quanto a suspensão da terapia imunossupressora de pacientes com doenças autoimunes e inflamações crônicas como psoríase, dermatite atópica, lúpus, esclerodermia e hidradenite supurativa que necessitam dessa terapia medicamentosa.

Contudo, para Rademaker et al., ${ }^{17}$ pacientes com problemas inflamatórios na pele e em tratamento com imunomoduladores devem interromper essa terapia ao ser diagnosticado com COVID-19, com exceção dos corticoides sistêmicos, devendo a conduta ser individualizada, tendo em vista que o indivíduo pode ser suscetível a uma infecção secundária e a complicações no quadro de COVID-19 que pode ser agravada pela imunossupressão. É importante cessar o tratamento da imunossupressão por pelo menos quatro semanas ou até que se reverta completamente o quadro de COVID-19.

A maioria dos estudos mostra que as manifestações cutâneas mais encontradas em pacientes confirmados com COVID-19, ou suspeitos, são as lesões acrais, principalmente nas extremidades do corpo, sem associação com exposição ao frio ou associação com outra doença. Esses indivíduos normalmente tiveram contato com portadores do vírus, o que pode ser importante para avaliação epidemiológica, pois eles podem ser possíveis transmissores. Porém, ainda requer muito estudo, observação e dados mais detalhados. ${ }^{18}$

TABELA 3: Manifestações clínicas encontradas em pacientes com casos suspeitos e confirmados de COVID-19 em estudos publicados em 2020.

\begin{tabular}{|c|c|c|c|}
\hline TÍTULO ARTIGO & AMOSTRA & $\begin{array}{l}\text { MANIFESTAÇÕES } \\
\text { CUTÂNEAS }\end{array}$ & OUTROS SINTOMAS \\
\hline $\begin{array}{l}\text { Cutaneous manifestations in COVID-19: } \\
\text { a first perspective }{ }^{9}\end{array}$ & 88 & $\begin{array}{l}\text { Erupções } \\
\text { eritematosas }\end{array}$ & $\begin{array}{c}\text { Febre, tosse seca, dispneia, rinorreia, } \\
\text { ageusia e anosmia }\end{array}$ \\
\hline $\begin{array}{c}\text { Chilblainlike lesions during COVID-19 epidemic: } \\
\text { a preliminary study on } 63 \text { patients }^{12} \\
\end{array}$ & 63 & Eritema pernioso & $\begin{array}{c}\text { Dor e prurido, Gastrointestinais, } \\
\text { Respiratórios e Febre }\end{array}$ \\
\hline $\begin{array}{l}\text { Chilblain-like lesions in pediatrics dermatological } \\
\text { outpatients during the COVID-19 outbreak }{ }^{14}\end{array}$ & 27 & $\begin{array}{l}\text { Lesões purpúricas } \\
\text { acro isquêmicas }\end{array}$ & Prurido e dor suave \\
\hline
\end{tabular}

\section{CONSIDERAÇÕES FINAIS}

A pandemia de COVID-19 vem afetando todo o mundo, porém a sintomatologia ainda é bastante incerta, já que o coronavirus não se manifesta apenas com sintomas respiratórios, como pneumonia e Síndrome Respiratória Aguda (SARS). Existem ainda 
diversos sinais e sintomas em estudo que podem ser associados a este vírus, como as manifestações dermatológicas.

Concluiu-se que algumas lesões cutâneas podem ser respostas de reações imunológicas, caracterizando-se, assim, como manifestações tardias de COVID-19. O aparecimento de lesões acroisquêmicas, semelhantes ao eritema pernioso e manifestações cutâneas relacionadas à vasculopatia, podem anteceder sintomas pulmonares da doença.

A estratificação dos pacientes portadores de manifestações cutâneas relativas ao COVID-19 tem grande impor- tância, a fim de que sejam identificados potenciais transmissores assintomáticos. Dermatologistas, pediatras e clínicos devem estar cientes das lesões associadas a infecção do COVID-19 e suas possíveis complicações.

Manifestações dermatológicas do COVID-19 geralmente são autolimitadas e ainda não se sabe ao certo o seu mecanismo, por isso são necessários estudos a fim de elucidar melhor os mecanismos dermatológicos do COVID-19, bem como, avaliar o valor preditor de relações dermatológicas precoces, ou não, diagnóstico com a gravidade da doença e um melhor manejo desses pacientes.

\title{
DERMATOLOGIC MANIFESTATIONS IN PATIENTS WITH COVID-19
}

\section{ABSTRACT}

COVID-19, a disease caused by the new coronavirus, SARS-Cov 2, emerged in China in December 2019 and quickly began to spread worldwide. However, the symptoms are very nonspecific and variable, making the clinical diagnosis of the disease difficult. Therefore, the present study aims to analyze the occurrence of skin manifestations and their types in patients with COVID-19. This is a literature review, conducted in October 2020, which guiding question was <Can dermatological manifestation in patients with COVID-19 be a diagnostic method for a patient with coronavirus? And what manifestations and complications are related to COVID-19?>. It was only in March 2020 that the first studies on dermatological manifestations were published, showing that the majority of patients do not have skin lesions and those who do have a maritally erythematous rash. However, the most different types of injuries have been reported. Owing to the difficulty in monitoring patients with coronavirus infections, its mechanism is not yet known, what is known is that the condition is self-limiting and that some drugs can induce its formation, making it difficult to differentiate between disorder and treatment. Therefore, the conducts must be individualized in order to bring a better therapeutic response and understanding of the pathology. It is important to be especially attentive to the sick who use immunosuppressive therapy.
\end{abstract}

KEYWORDS: Coronavirus. Skin Manifestations. Coronavirus Infections. 


\section{REFERÊNCIAS}

1. Nobre AFS, Sousa RCM, Santos M C, Barbagelata LS, Costa Júnior E, Lima DFa et al - Primeira detecção de coronavírus humano associado à infecção respiratória aguda na Região Norte do Brasil. Rev Pan-Amaz Sau. 2014; 5( 2 ): 37- 41 .

2. Organização Pan-Americana da Saúde (BR). Folha informativa - COVID-19 (doença causada pelo novo coronavírus) [ acesso em 20 de out 2020]. Disponivel em: <https://www.paho.org/ pt/topicos/coronavirus/doenca-causada-pelonovo-coronavirus-covid-19>.

3. Organização Pan-Americana da Saúde (BR). Folha informativa COVID-19. [ acesso em 21 de out 2020]. Disponivel em: < https://www.paho. org/pt/covid19>.

4. Zhang JJ, Dong X, Cao YY, et al. Clinical characteristics of 140 patients infected with SARS-CoV-2 in Wuhan, China. Allergy. 2020; 75(7):1730-41.

5. Young S, Fernandez AP. Skin manifestations of COVID-19. Cleve Clin J Med. 2020 june, DOI: https://doi.org/10.3949/ccjm.87a.ccc031.

6. Zhang Y, Cao W, Xiao M, et al. Características clínicas e de coagulação de 7 pacientes com pneumonia crítica por COVID - 2019 e acro isquemia . Zhonghua Xue Ye Xue Za Zhi . 2020; 41(4): 302-07.

7. Thachil J, Tang N, Gando S, et al. ISTH interim guidance on recognition and management of coagulopathy in COVID-19. J Thromb Haemost. 2020;18(5):1023-26.

8. Kannan S, Shaik Syed Ali P, Sheeza A, Hemalatha K. COVID-19 (Novel Coronavirus 2019) - recent trends. Eur Rev Med Pharmacol Sci. 2020; 24(4): 2006-11.
9. Recalcati, S. Cutaneous manifestations in COVID-19: a first perspective. Jour Europ Acad Dermat and Vener. 2020; (34): 213-13.

10. Gupta A, Madhavan M V, Sehgal K, Nair N, Mahajan S, Sehrawat TS, Freedberg, DE. Extrapulmonary manifestations of COVID-19. Nat med. 2020; 26(7), 1017-32.

11. Daneshgaran G, Dubin DP, Gould DJ. Cutaneous Manifestations of COVID-19: An Evidence-Based Review. Am J Clin Dermatol. 2020; 21(5): 627-39. doi:10.1007/\$40257-02000558-4

12. Piccolo V, Neri I, Filippeschi C, Oranges T, Argenziano G, Battarra VC, et al. Chilblainlike lesions during COVID-19 epidemic: a preliminary study on 63 patients. Journal of the European Academy of Dermatology and Venereology: JEADV. 2020; (34): e291-e345

13. de Masson A, Bouaziz JD, Sulimovic L, Cassius C, Jachiet M, Ionescu MA, Rybojad M, Bagot $M$, Duong TA; SNDV (French National Union of Dermatologists-Venereologists). Chilblains is a common cutaneous finding during the COVID-19 pandemic: A retrospective nationwide study from France. J Am Acad Dermatol. 2020; 83(2): 667-70.

14. Garcia-Lara G, Linares-González L, RódenasHerranz T, Ruiz-Villaverde R. Chilblain-like lesions in pediatrics dermatological outpatients during the COVID-19 outbreak. Dermatologic Therapy. 2020; 33 (5): 13516-520.

15. ia JL, Kamceva M., Rao SA, Linos E. Cutaneous manifestations of COVID-19: a preliminary review. Jour Amer Acad Dermat. 2020; 83(2), 687-90.

16. Darlenski R., Tsankov N. Covid-19 pandemic and the skin-What should dermatologists know?. 
Clinics in Dermatology.. 2020; 61(2): 158-59.

17. Rademaker $M$, Baker C, Foley $P$, Sullivan, J, Wang C. Advice regarding COVID-19 and use of immunomodulators, in patients with severe dermatological diseases. Aust. J. Dermatol. 2020; 61(2): 158-59.
18. Witkowski A, Ludzik J, Hansel DE, Raess PW, White K, Leachman S. Case Report: Chilblainslike lesions (COVID-19 toes) during the pandemic - is there a diagnostic window? F1000Research. 2020; 9: 668-673 